\title{
Research of Contact Response of an Elastic Sphere Impacting a Rigid Plate Based on the Mass-Spring- Damped Oscillator Model and Dimensionless Parameter $\omega_{\Omega}$
}

\author{
Yang Yang, Qingliang Zeng ${ }^{\dagger}$, Lirong Wan, Liang Wang and Guangjun Yin \\ Department of Mechanical and Electrical Engineering, ShanDong University of Science and Technology, 266590, \\ QingDao, ShanDong, China. \\ $\dagger$ Corresponding author
}

\begin{abstract}
(Received 25 May 2018; accepted 10 December 2018)
The contact theory, such as the Hertz theory and Hunt-Crossley model can just solve the contact response when the sphere goes to the maximum compression but can't solve the contact process. In traditional research the impact process is considered as a single degree of freedom dynamical spring-damping system, however, the calculated contact force is much greater than the actual one. Based on the shortage of the conducted studies, a new dimensionless parameter $\omega_{\Omega}$ is proposed in this paper, the mass-spring-damped oscillator model (MSDOM) and the new contact theory with the consideration of the sphere mass and $\omega_{\Omega}$ are established. The correctness of the theoretical model is verified by 3D simulation, and the optimal contact force model is obtained. Furthermore, influence rules of material properties and contact parameters on contact response were studied. The results show that the contact deformation, velocity, piecewise nonlinear contact force and acceleration based on MSDOM and $\omega_{\Omega}$ are effective to calculate the contact response. The contact stiffness, damping, material properties of sphere and elastic modulus of the plate have different influence on the contact response while the Poisson's ratio $\nu_{2}$ has little effect on it.
\end{abstract}

\section{INTRODUCTION}

Interface contact is a classical mechanical behavior that occurs in various fields, ${ }^{1-4}$ such as in bullet shooting, axle head and sleeve, multi-body system and so on. The contact mechanism and contact response between objects is different under different contact conditions. Taking coal gangue impacting metal plate of tail beam of the caving coal hydraulic support as an example, the coal gangue and the metal plate can be equivalent to elastic sphere particles with the properties of coal or gangue, and rigid plate respectively for the reason that the elastic modulus of coal or gangue are much smaller than that of the metal plate. Due to the different angles and velocities of impact between the coal gangue and the metal plate, we studied the working condition of an elastic sphere impacting the rigid plate vertically.

Equivalent elastic Hertz contact theory is a common approach for solving contact problems between spheres or sphere and plane.$^{5-10}$ In the case of the known contact deformation, we can get the contact force, stress and other related parameters through the Hertz contact theory, and combined with the law of conservation of energy, we can get the maximum contact deformation and maximum contact force. Based on the Hertz contact theory, Hunt-Crossley, Lankarani-Nikravesh and Flores ${ }^{11-14}$ established different types of contact theories that considered the energy consumption mechanism, and damping energy of the system was taken into account, which can calculated the contact force and other contact parameters more accurately. However, these contact theories only established the relationship between contact force and contact deformation or contact force and contact deformation, velocity, and recovery coefficient. For conditions of elastic sphere impact- ing rigid plate, the instantaneous velocity and instantaneous contact compression of the elastic sphere are unknown parameters. The impact contact process cannot be calculated and solved based on the above theory. BISCHOFF et al. proposed a simple spring-mass model to calculate the contact force-time relationship ${ }^{15}$ in 1990 , but they did not consider the damping energy of the system. Qin Zhiying et al. used the single-degree-of-freedom dynamical spring-damping model as the equivalent of the impact contact process, ${ }^{16}$ and the linear damping model $^{17}$ was used to solve the linear damping model, but the impact of mass on the impact contact was not considered. Huifang Xiao et al. established the general equation of motion for the free vertical oscillations of a mass hanging on a nonlinear spring ${ }^{18}$ based on nonlinear stiffness but did not consider the effect of damping on the system balance.

For small mass particles impacting rigid plates, due to the small particle size and the small contact force generated by low impact velocity, the particle mass and system damping are not negligible factors. Based on these analyses, this paper established MSDOM which considered mass, contact stiffness and damping. In combination with the simulation of LSDYNA, dimensionless parameter $\omega_{\Omega}$ was used instead of the system's undamped natural frequency $\omega_{n}$ to obtain the models of contact displacement and velocity theory based on MSDOM and dimensionless parameter $\omega_{\Omega}$. By comparing the simulation with the MSDOM and the piecewise nonlinear contact force model, it could be known that the piecewise nonlinear contact force model has more advantages and based on this to obtain the piecewise nonlinear acceleration theory model. On this foundation, we studied the influence of different contact parameters and material properties on impact contact response.

The remainder of the paper is organized as follows: Section 


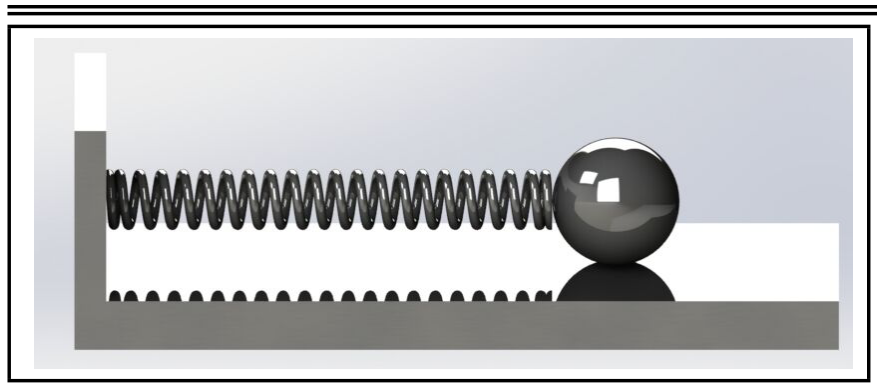

Figure 1. A simple spring oscillator model.

2 establishes the MSDOM based on dimensionless parameter $\omega_{\Omega}$. Section 3 introduces the method of constructing the simulation model, compares the analysis result of theory with simulation, determines the value of nonlinear order $\Omega$, and determines the nonlinear contact force theory model as the optimal contact force model. Section 4 analyses the effect of contact parameters and material properties on contact response. Section 5 shows some related work and our conclusions.

\section{THE MSDOM BASED ON DIMENSI- ONLESS PARAMETER $\omega_{\Omega}$}

\subsection{Hertz Contact}

According to Hertz contact theory, ${ }^{19}$ the relationship between the contact force $\mathrm{P}$ and contact deformation $\gamma$ in the impacting progress of the two sphere is:

$$
\begin{gathered}
P=k \cdot \delta^{n} ; \\
k=\frac{4}{3}\left(\frac{1-\nu_{1}^{2}}{E_{1}}+\frac{1-\nu_{2}^{2}}{E_{2}}\right)^{-1} \cdot\left(\frac{R_{1} R_{2}}{R_{1}+R_{2}}\right)^{\frac{1}{2}} ;
\end{gathered}
$$

where: $n$-The nonlinear exponent, $n=3 / 2$ in Hertz contact theory. $E_{i}$ - Elastic modulus, for the two spheres, $i=1, i=2$ respectively. $\nu_{i}$ - Poisson's ratio. $R_{i}$ - The contact curvature radius of two contactants at the collision point.

For the impact between the sphere and plate, at the same time, sphere $i=1$ and plate $i=2$, the contact curvature radius of the plate $R_{2} \rightarrow \infty$, the contact stiffness $k_{1}=\frac{4 \sqrt{R_{1}}}{3}\left(\frac{1-\nu_{1}^{2}}{E_{1}}+\frac{1-\nu_{2}^{2}}{E_{2}}\right)^{-1}$ then:

$$
P=k_{1} \cdot \delta^{n}=\frac{4 \sqrt{R_{1}}}{3}\left(\frac{1-\nu_{1}^{2}}{E_{1}}+\frac{1-\nu_{2}^{2}}{E_{2}}\right)^{-1} \cdot \delta^{n} .
$$

\subsection{The Mass-Spring-Damped Oscillator Model (MSDOM)}

According to Hooke's Law, as is shown in Fig. 1, the relation between the elastic force $\mathrm{F}$ in the motion process of the object and the relative displacement $x$ of the object to the equilibrium position is:

$$
F=k x, \frac{d^{2} x}{d t^{2}}=-\frac{k}{m} x=-\omega^{2} x .
$$

The vibration frequency is given by:

$$
\omega=\sqrt{\frac{k}{m}} .
$$

The traditional vertical free mass collision model does not consider the gravity of the object. Because of the small size of

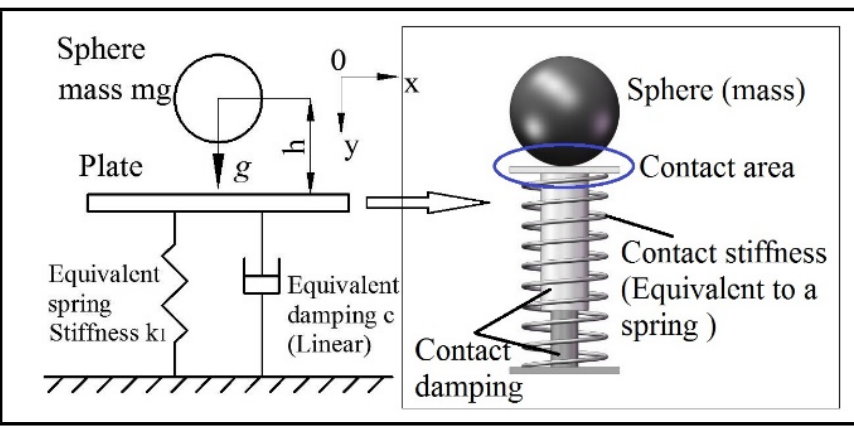

Figure 2. The MSDOM.

the particles in this paper, the collision contact force produced by the small impact velocity is limited, and the influence of the particles own gravity on the system impact response cannot be directly ignored. Based on the influence of particles own gravity, a mass-spring-damped oscillator model (MSDOM) shown in Fig. 2 is established:

$$
\left\{\begin{array}{l}
m \ddot{y}(t)=m g-c \dot{y}(t)-k_{1} y(t) \\
y\left(t_{0}\right)=0 \\
\dot{y}\left(t_{0}\right)=v_{0} \\
v_{0}=\sqrt{2 g h}
\end{array} ;\right.
$$

where $c$ is the contact damping between the sphere and the plate, $k_{1}$ is the contact stiffness between the sphere and the plate.

The theoretical model of vibration response is obtained after Laplace numerical transformation and inverse transformation:

$$
\begin{aligned}
y(t)=\frac{g}{\omega_{n}^{2}}\left(1-e^{-\xi \omega_{n} t} \cdot \cos \omega_{d} t\right) & \\
& +\frac{\omega_{d} v_{0}-g \xi \sqrt{1-\xi^{2}}}{\omega_{d}^{2}} e^{-\xi \omega_{n} t} \cdot \sin \omega_{d} t
\end{aligned}
$$

which may be written in the form:

$$
y(t)=A_{1}\left(1-e^{-\xi \omega_{n} t} \cos \omega_{d} t\right)+A_{2} e^{-\xi \omega_{n} t} \sin \omega_{d} t
$$

where $\quad A_{1}=\frac{g}{\omega_{n}^{2}} ; \quad A_{2}=\frac{\omega_{d} v_{0}-g \xi \sqrt{1-\xi^{2}}}{\omega_{d}^{2}} ; \quad \xi=\frac{c}{2 m \omega_{n}}<1$; $\omega_{d}=\sqrt{1-\xi^{2}} \omega_{n} ; \omega_{n}=\sqrt{\frac{k_{1}}{m}}$.

We derive the acceleration equation after second order derivation for Eq. (8),

$$
\ddot{y}(t)=B_{1} e^{-\xi \omega_{n} t} \sin \left(\omega_{d} t+\arctan B_{2}+\pi\right) ;
$$

with:

$$
\begin{gathered}
B_{1}=\sqrt{\left(g-2 \xi v_{0} \omega_{n}\right)^{2}+\frac{\left(2 \xi^{2} v_{0} \omega_{n}-g \xi-v_{0} \omega_{n}\right)^{2}}{\left(1-\xi^{2}\right)}} ; \\
B_{2}=\frac{\left(g-2 \xi v_{0} \omega_{n}\right) \sqrt{1-\xi^{2}}}{2 \xi^{2} v_{0} \omega_{n}-g \xi-v_{0} \omega_{n}} .
\end{gathered}
$$

From the condition $F(t)=c \dot{y}+k y=0$ and $\ddot{y}(\Delta t)=g(\Delta t$ is the collision contact time):

$$
\ddot{y}(\Delta t)=B_{1} e^{-\xi \omega_{n} t} \sin \left(\omega_{d} \Delta t+\arctan B_{2}+\pi\right) .
$$


The order of magnitude for $B_{1}$ is $10^{5}, \frac{g}{B_{1}} \approx 0$, then:

$$
\omega_{d} \Delta t+\arctan B_{2}+\pi=k \pi
$$
lows:

Hence, the collision contact time can be expressed as fol-

$$
\Delta t=\frac{1}{\omega_{d}}\left(\pi-\arctan B_{2}\right) .
$$

The derivation of Eq. (3) is obtained:

$$
\dot{y}(t)=C_{1} e^{-\xi \omega_{n} t} \cos \left(\omega_{d} t-\arctan C_{2}\right) ;
$$

where: $C_{1}=\frac{1}{\omega_{n}} \sqrt{\frac{g^{2}-2 g \xi v_{0} \omega_{n}+v_{0}^{2} \omega_{n}^{2}}{1-\xi^{2}}}, C_{2}=\frac{g-\xi v_{0} \omega_{n}}{v_{0} \omega_{n} \sqrt{1-\xi^{2}}}$.

Take the Eq. (14) to Eq. (15), we obtain the velocity of the sphere at collision ended time:

$$
\begin{aligned}
& v_{2}=\dot{y}(\Delta t)= \\
& C_{1} \exp \left[\frac{-\xi}{\sqrt{1-\xi^{2}}}\left(\pi-\arctan B_{2}\right)\right] \cos \left(\pi-\arctan D_{1}\right)
\end{aligned}
$$

with: $D_{1}=\frac{-\xi v_{0}^{2} \omega_{n}^{2}-g^{2} \xi+2 g \xi^{2} v_{0} \omega_{n}}{\sqrt{1-\xi^{2}}\left(-v_{0}^{2} \omega_{n}^{2}+2 g \xi v_{0} \omega_{n}-g^{2}\right)}$.

If the corresponding time of the maximum contact displacement of the sphere is $t_{c}, t_{c}<\Delta t$. Take $t_{c}$ to Eq. (15), $\dot{y}\left(t_{c}\right)=0$, it can be rewritten as:

$$
\begin{gathered}
C_{1} e^{-\xi \omega_{n} t_{c}} \cos \left(\omega_{d} t_{c}-\arctan C_{2}\right)=0 \\
t_{c}=\frac{1}{\omega_{d}}\left(\frac{\pi}{2}+\arctan C_{2}\right) .
\end{gathered}
$$

Take Eq. (18) to Eq. (8), the maximum contact displacement is:

$$
y\left(t_{c}\right)=A_{1}\left(1-e^{-\xi \omega_{n} t_{c}} \cos \omega_{d} t_{c}\right)+A_{2} e^{-\xi \omega_{n} t_{c}} \sin \omega_{d} t_{c} .
$$

The recovery coefficient $e_{h}$ in the process of system collision can be expressed as:

$$
\begin{array}{r}
e_{h}=-\frac{v_{2}}{v_{0}}=-\frac{C_{1}}{v_{0}} \exp \left[\frac{-\xi}{\sqrt{1-\xi^{2}}}\left(\pi-\arctan B_{2}\right)\right] \\
\cdot \cos \left(\pi-\arctan D_{1}\right)
\end{array}
$$

and the contact force:

$$
F[y(t)]=m g-m B_{1} e^{-\xi \omega_{n} t} \sin \left(\omega_{d} t+\arctan B_{2}+\pi\right) .
$$

\subsection{The MSDOM Based on Dimensionless Parameter $\omega_{\Omega}$}

Through the establishment of the MSDOM and its theoretical solution of the contact force, contact deformation, velocity and accelerated velocity (Eq. (7), Eq. (9), Eq. (15) and Eq. (21)), we obtained the contact response of the system in which its elastic contact force and contact damping was linear.
As shown in Fig. 3, in the contact system of coal or gangue particles impact on metal plate, coal or gangue particles were equivalent to the elastic sphere and the metal plate are equivalent to a rigid plate. The system damping in the contact process was not the simple linear damping. On this occasion, the contact response gets from the MSDOM (For example, contact force, contact deformation, velocity and accelerated velocity get from Eq. (7), Eq. (9), Eq. (15) and Eq. (21)) based on linear damping is inaccurate.

Hertz et al. has already proved that the elastic contact force in the contact process isn't linear. From Eq. (1), the contact force defined by Hertz is $P=k \cdot \delta_{n}$, where $n$ is the nonlinear order of the contact deformation $\delta$. Under the linear damping, the equation of the contact force, contact deformation, velocity and accelerated velocity is related to the natural frequency $\omega_{n}$. To determine the form of nonlinear contact force, in combination with the form of the Hertz contact force, we generalise and defined the dimensionless parameter $\omega_{\Omega}=\omega_{n} \frac{1}{\Omega}(\Omega$ is the nonlinear order of the natural frequency $\omega_{n}, \Omega$ are different for different contact conditions. According to different materials and contact conditions, we can determine the value of nonlinear order $\Omega$, and get the exact contact force theoretical model.), then $\omega_{\Omega}=\left(\frac{k_{1}}{m}\right)^{\frac{1}{2 \Omega}}$. Replace the $\omega_{n}$ and $\omega_{d}$ by $\omega_{\Omega}$ and $\omega_{d \Omega}$ and substituting $\omega_{\Omega}$ and $\omega_{d \Omega}$ to the mass-spring-damped oscillator dynamic model. The contact model can be expressed as:

$$
\left\{\begin{aligned}
y_{\Omega}(t)= & \frac{g}{\omega_{\Omega}^{2}}\left(1-e^{-\xi \omega_{\Omega} t} \cos \omega_{d \Omega} t\right)+ \\
& \frac{\omega_{d \Omega} v_{0}-g \xi \sqrt{1-\xi^{2}}}{\omega_{d \Omega}^{2}} e^{-\xi \omega_{\Omega} t} \sin \omega_{d \Omega} t \\
\dot{y}_{\Omega}(t)= & C_{1 \Omega} e^{-\xi \omega_{\Omega} t} \cos \left(\omega_{d \Omega} t-\arctan C_{2 \Omega}\right) \\
F[y(t)]= & m g-m B_{1 \Omega} e^{-\xi \omega_{\Omega} t} \\
& \cdot \sin \left(\omega_{d \Omega} t+\arctan B_{2 \Omega}+\pi\right)
\end{aligned}\right.
$$

$$
\begin{aligned}
& e_{h \Omega}=-\frac{v_{2}}{v_{0}}=-\frac{C_{1 \Omega}}{v_{0}} \\
& \cdot \exp \left[\frac{-\xi}{\sqrt{1-\xi^{2}}}\left(\pi-\arctan B_{2 \Omega}\right)\right] \cos \left(\pi-\arctan D_{1 \Omega}\right) ;
\end{aligned}
$$

$$
\begin{array}{r}
y_{\Omega}\left(t_{c \Omega}\right)=A_{1 \Omega}\left(1-e^{-\xi \omega_{\Omega} t_{c \Omega}} \cos \omega_{d \Omega} t_{c \Omega}\right) \\
+A_{2 \Omega} e^{-\xi \omega_{\Omega} t_{c \Omega}} \sin \omega_{d \Omega} t_{c \Omega} .
\end{array}
$$

Combine with the Hunt-Crossley contact model, ${ }^{11}$ we obtain the new nonlinear contact force model:

$$
\begin{aligned}
& F[y(t)]=k_{1} y_{\Omega}(t)^{n} \\
& \cdot\left[1+\frac{3\left(1-e_{h \Omega}\right) C_{1 \Omega} e^{-\xi \omega \Omega t} \cos \left(\omega_{d \Omega} t-\arctan C_{2 \Omega}\right)}{2 v_{0}}\right]
\end{aligned}
$$

Based on this, a piecewise nonlinear contact force model $^{20,21}$ is extended as follows: 


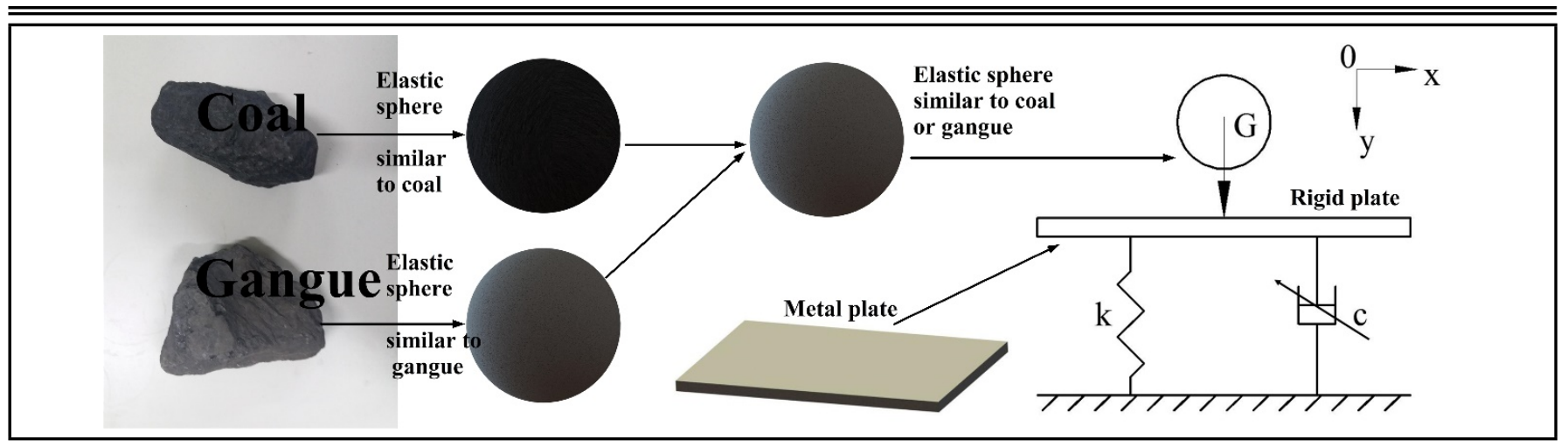

Figure 3. Elastic impact model of coal or gangue partical on the rigid metal plate.

$$
\begin{aligned}
& F[y(t)]= \\
& \left\{\begin{array}{cl}
k_{1} y_{\Omega}(t)^{n} & \\
\cdot\left[1+\frac{3\left(1-e_{h \Omega}\right) \dot{y}_{\Omega}(t)}{2 v_{0}}\right], & y(t) \geq 0, \dot{y}(t)>0 \\
k_{2}\left(y_{\Omega}(t)-y_{\Omega}(0)\right)^{n} & \\
\cdot\left[1+\frac{3\left(1-e_{h \Omega}\right) \dot{y}_{\Omega}(t)}{2} v_{0}\right], & y(t) \geq y(0), \dot{y}(t)<0 \\
0, & \text { Others }
\end{array}\right.
\end{aligned}
$$

where $y_{\Omega}(0)=\left(1-e_{h \Omega}^{2}\right) y\left(t_{c \Omega}\right) ; k_{1} / k_{2}$-Contact stiffness in compression stage and recovery phase, $k_{2}=\frac{1}{e_{h \Omega}^{2 n}} k_{1}$.

\section{3D NUMERICAL SIMULATION BY LSDYNA}

\subsection{Simulation Model}

Simulation of coal or gangue particle vertical impact the metal plate was carried out on the Lsdyna platform. Establish the model of spherical coal gangue. To simulate the impacted rigid surface, we defined the material type of metal plate as a rigid body. In order to simulate the impact load better, the acceleration of the sphere was defined as $9.8 \mathrm{~m} / \mathrm{s}^{2}$ to simulate earth's gravity, grids of the contact area between the metal plate and sphere were meshed. If the initial height between coal or gangue and the upper surface of metal plate is $3 \mathrm{~m}$, the gap between the end point of sphere and the upper end-face of the metal plate was defined to be $0.001 \mathrm{~m}$ in the simulation model establish process and the initial velocity of sphere is $7.668 \mathrm{~m} / \mathrm{s}$ to shorten the calculation time. The effect of the gap can be negligible, but it is remarkable to prevent the sphere from overlapping with the mesh of metal plate.

In this paper, we mainly studied the vertical impact contact response between elastic sphere and target plate in elastic half-space. In the process of modeling, the target plate was set as the thin metal plate with homogeneous continuous medium and the sphere models was set as the viscoelastic constitutive models with the properties of rock (coal or gangue). The contact type was set to the Automatic (ASSC) in the type of Surface to Surf. The surface of the sphere was set as the contact surface and the surface of the metal plate was the target surface. The constraint is applied to four corners of the metal plate, and the constraint type is set as full constraint. The simulation model is shown in Fig. 4.

It mainly studies the elastic contact property between elastic particle which has similar properties to coal or gangue and

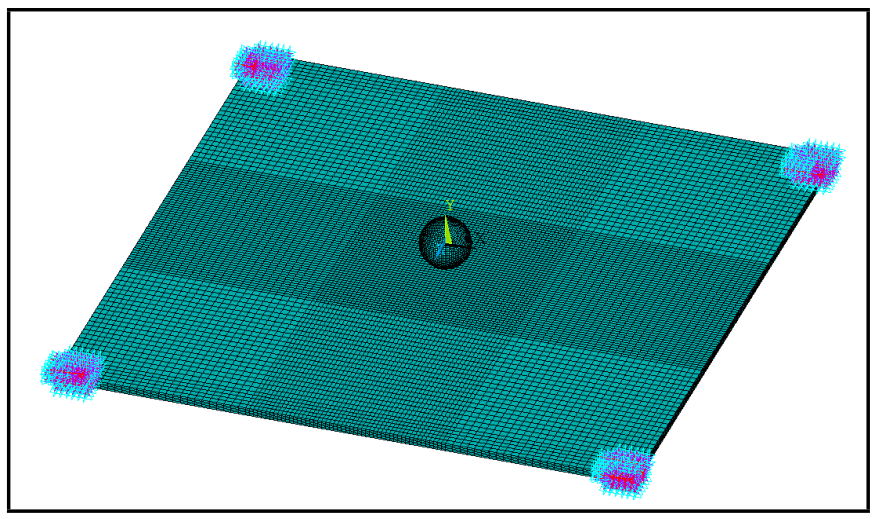

Figure 4. Simulation model.

Table 1. Parameters of contact model of elastic sphere and metal plate

\begin{tabular}{|c|c|c|c|c|c|}
\hline $\begin{array}{c}\text { Material of } \\
\text { contactant }\end{array}$ & $\begin{array}{c}\rho \\
\left(\mathrm{kg} \cdot \mathrm{m}^{3}\right)\end{array}$ & $\begin{array}{c}E \\
(\mathrm{GPa})\end{array}$ & $\nu$ & $\begin{array}{c}\text { Size } \\
(\mathrm{mm})\end{array}$ & $\begin{array}{c}\text { Material } \\
\text { type }\end{array}$ \\
\hline Coal & 1380 & 2.26 & 0.28 & $\mathrm{R} 25$ & Elastic \\
\hline Gangue & 1550.6 & 3.906 & 0.28 & $\mathrm{R} 25$ & Elastic \\
\hline Metal plate & 7850 & 210 & 0.30 & $600 \times 580 \times 8$ & Rigid \\
\hline
\end{tabular}

rigid plate which has similar properties to the metal plate. In the process of establishing the simulation model, defined the material type of spherical coal or gangue as elastic mate-rials. The model parameters of the sphere and the metal plate are shown in Table 1.

\subsection{Determination of Nonlinear Order $\Omega$}

The contact deformation and contact force of the elastic sphere which has similar properties to coal impacting metal plate are obtained through the simulation, the thick solid red lines are the simulation curves of the contact force and contact deformation, as is shown in Fig. 5 and Fig. 6 respectively. In order to determine the value of nonlinear order in dimensionless parameter $\omega_{\Omega}$ of the system, and then determine the theoretical solution of the contact force, we took $\Omega=1: 0.05: 1.4$ respectively. Theoretical contact forces and contact deformations under different $\Omega$ were shown by dotted lines with different colours in the figures.

The theoretical maximum contact deformation increased with the increase of $\Omega$, and the theoretical maximum contact force and vibration frequency decreased gradually. The theoretical curve of the contact force was highly consistent with the simulation curve when $\Omega=1.247$ or $\Omega=1.25$, the theoretical curve of contact deformation was also highly consistent with the simulation when $\Omega=1.247$. The deviation between the theoretical curve and the simulation curve was greater when 


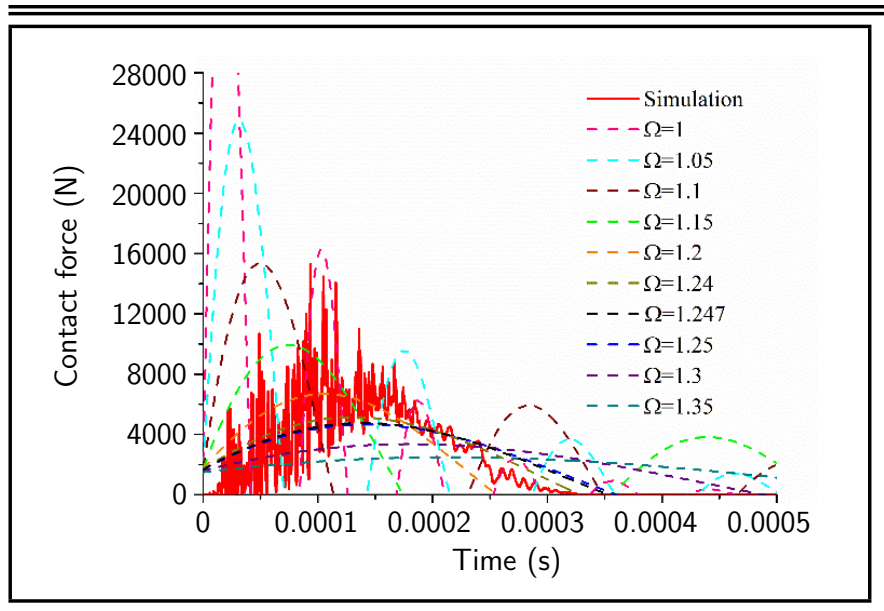

Figure 5. Curves of contact force-time.

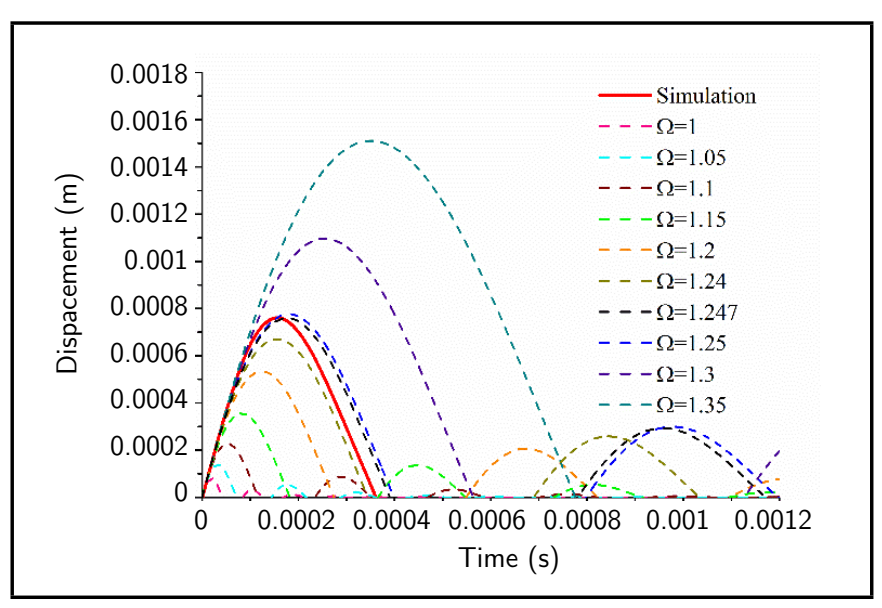

Figure 6. Curves of contact deformation-time.

$\Omega$ take different values. The nonlinear order $\Omega$ is 1.247 in the progress of an elastic sphere which had similar properties to coal impacting metal plate. When taking contact force and contact displacement as criteria, at the moment, $\omega_{\Omega}=\left(\frac{k}{m}\right)^{\frac{500}{1247}}$.

\subsection{Comparison and Analysis of Theoretical and Simulation Results}

The same volume of elastic spheres with properties of coal and gangue were used for impact simulation respectively, the simulation results could be obtained from Ls-prepost, and combined with the theoretical model that $\Omega=1.247$, the theoretical and simulation curves of displacement-time, velocitytime, acceleration-time and contact force-time of coal and gangues can be got in Figs. 7-10.

It can be known from Fig. 7 that the simulated displacements of coal and gangue is basically consistent with the theoretical displacements. The theoretical maximum displacement and simulated displacement of coal is $7.59656 \times 10^{-4} \mathrm{~m}$ and $7.55635 \times 10^{-4} \mathrm{~m}$ respectively, and the difference between them is $0.53 \%$. The theoretical maximum displacement and simulated displacement of gangue is $7.16554 \times 10^{-4} \mathrm{~m}$ and $7.28359 \times 10^{-4} \mathrm{~m}$ respectively, and the difference between them is $1.65 \%$. The maximum displacement of elastic sphere with similar properties to coal is greater than that of with similar properties to gangue, the reason is that in the impact process, the contact damping and contact stiffness of gangue are larger than coal and leads to the gangue's greater resistance to elastic deformation and less impact contact time than coal.

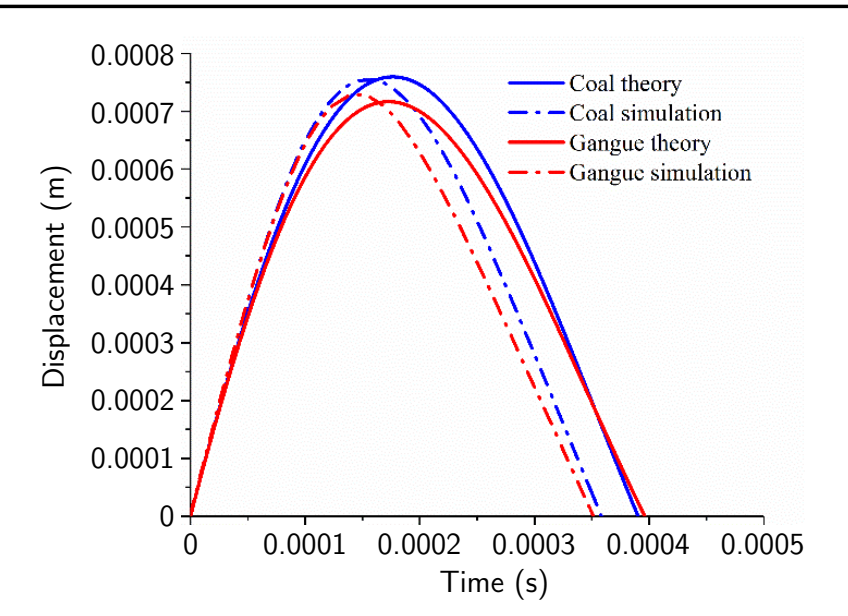

Figure 7. Contact deformation-time

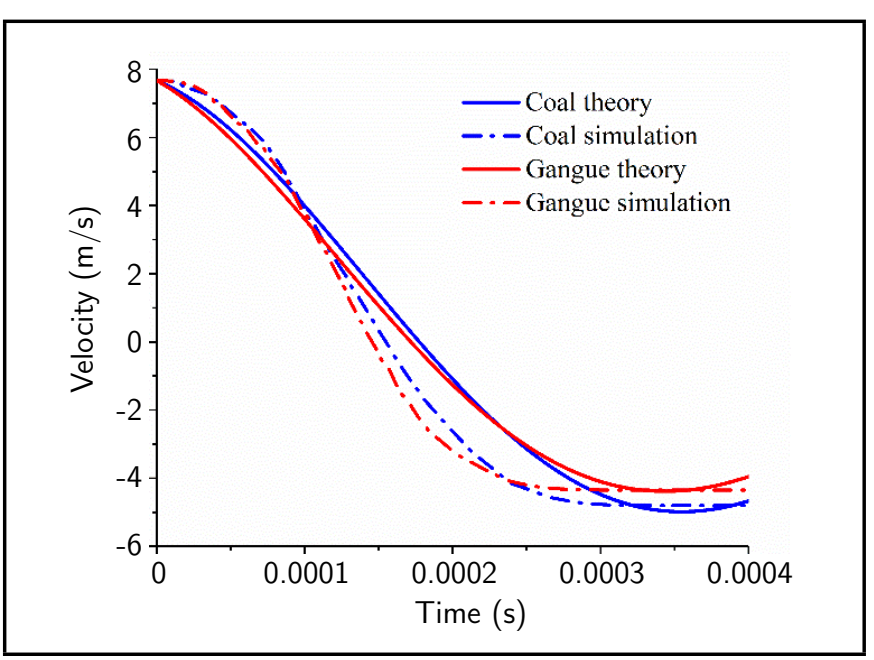

Figure 8. Velocity-time.

Fig. 8 shows that the velocity curve of coal in simulation has the same curve forms with the theoretical velocity curve and there are intersections between the simulation curve and the theoretical curve. The entire curves can be divided into two phases, the theoretical values in earlier contact stage are slightly lower than the simulation values, the theoretical values at the end of contact are slightly higher than the simulated values. The theoretical impact residual velocity and the simulated impact residual velocity of coal is $-4.98446 \mathrm{~m} / \mathrm{s}$ and $4.80414 \mathrm{~m} / \mathrm{s}$ respectively, and the difference between them is $3.75 \%$. The theoretical impact residual velocity and the simulated impact residual velocity of gangue is $-4.38497 \mathrm{~m} / \mathrm{s}$ and $-4.35128 \mathrm{~m} / \mathrm{s}$ respectively, and the difference between them is $0.77 \%$. There are large damping and stiffness in the process of impact contact between gangue and metal plates, and the energy consumed is greater than coal. Therefore, the residual kinetic energy of the unit mass of gangue is smaller than coal at the end of impact, causing the absolute value of the impact residual velocity of gangue is less than that of coal. It can be seen from the Fig. 9 and Fig. 10 that the simulation curves of contact force and contact acceleration exhibit oscillations apparently due to: (1) The randomness and uncertainty of the impact process; (2) For the elastic properties of the elastic sphere, the displacement of mesh in different positions of elastic sphere is variable in time and numerical; (3) When the elastic wall contacts with the rigid wall, a slight negative vol- 


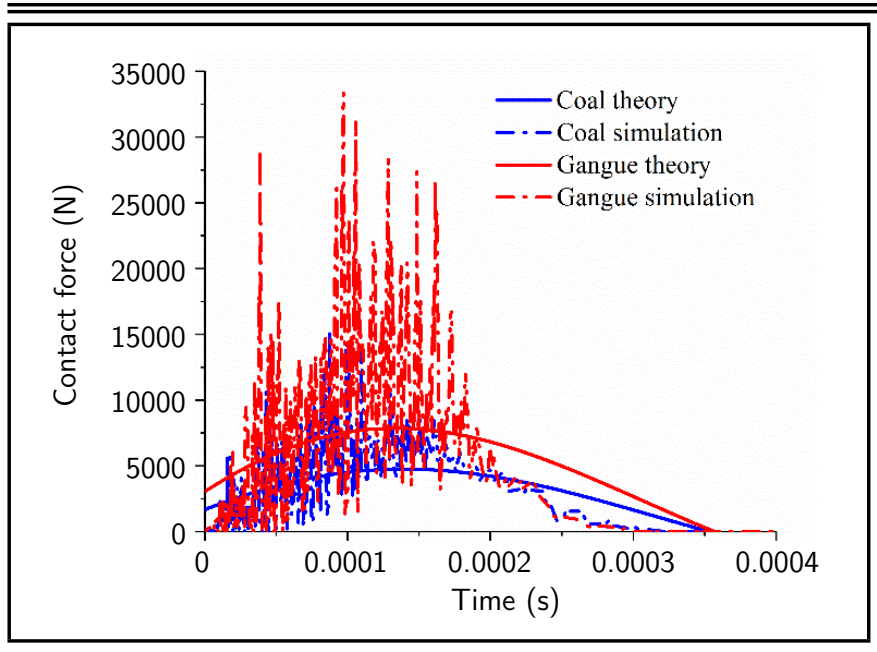

Figure 9. Contact force-time.

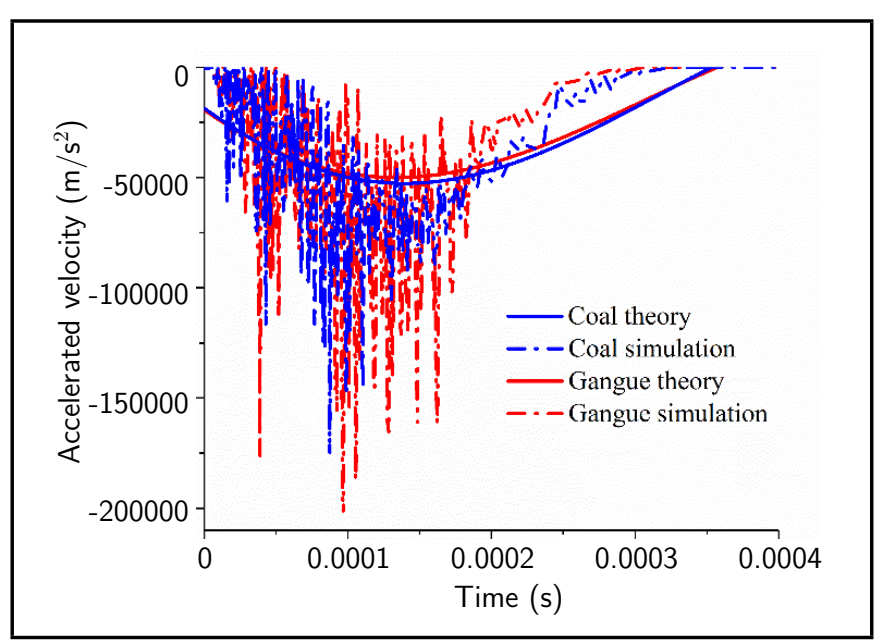

Figure 10. Accelerated velocity-time.

ume appears and causes fluctuations of the curves.

Figure 9 shows that the theoretical contact force curves all pass through the simulated contact force curves, and whole variant trend is identical. However, in the simulation process, the contact stiffness and contact damping in compression stage gradually increase from 0 to the maximum value (the maximum value is greater than the value defined by the linear theory), the contact stiffness and contact damping in recovery stage gradually decrease from the maximum value to 0 , resulting in contact force simulation results lower than the theoretical value first and then higher than the theoretical value, and then lower than the theoretical value. The maximum value of the contact force theory is far less than its simulation.

From Fig. 10, we could get that the acceleration curves of coal and gangue pass through the simulated acceleration curves, and whole variant trend is identical, but the average value of the simulated acceleration curves deviates greatly from the theoretical value.

From the foregoing, the linear displacement and velocity based on MSDOM and the dimensionless parameter $\omega_{\Omega}$ coincide with the simulation results, and the contact displacement, maximum contact displacement, impact velocity and impact residual velocity can be accurately predicted. The theoretical curves of linear acceleration and contact force based on MSDOM and dimensionless parameter $\omega_{\Omega}$ have the same overall trend with the simulation curve, but due to the setting of linear

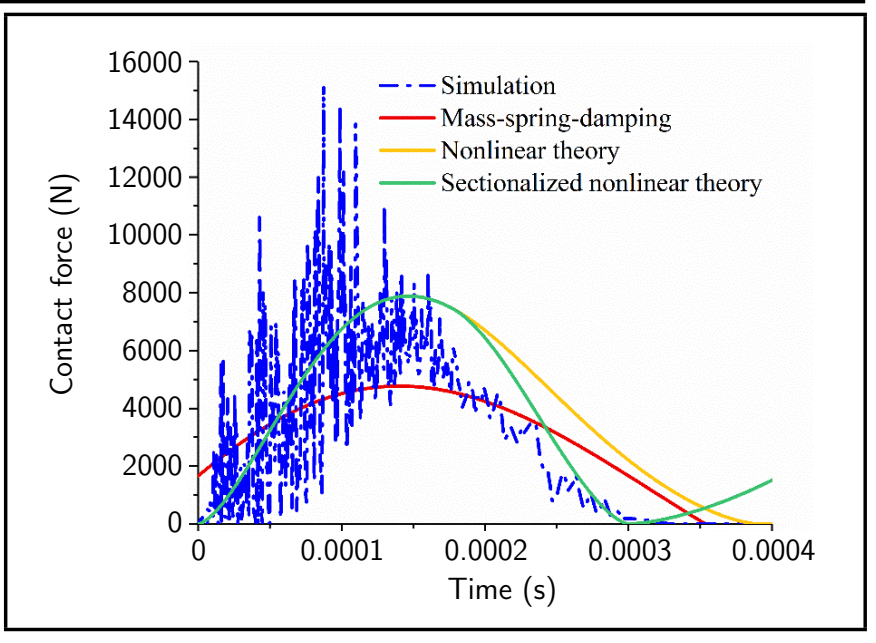

Figure 11. Curves of contact force-time.

damping in the theoretical derivation process. The average values of the simulated acceleration curves and simulated contact force curves deviate from the theoretical values.

\subsection{Optimal Contact Force Model}

The deviation between theoretical contact force obtained from MSDOM and the simulation value is bigger. Based on MSDOM, dimensionless parameter $\omega_{\Omega}$ and nonlinear contact force model, we obtain the different theoretical curves and simulation curve of contact force-time in the condition of an elastic sphere which has similar properties to coal impacting metal plate, as is shown in Fig. 11.

Due to the damping force $c \dot{y}(t)$ in the MSDOM force balance equation, the theoretical force of MSDOM is not started from 0 , and we cloud get that the peak value of the theoretical contact force obtained from MSDOM is larger than the average value of the simulation force from the above analysis and Fig. 11. The maximum value of the nonlinear contact force and the piecewise nonlinear contact force are the same, which are close to the peak value of the simulated force average value. However, the nonlinear contact force decreases slowly that leads to the long contact time and large deviation from the simulated contact force. Through comprehensive analysis of the results, we got that the piecewise nonlinear contact force curve has the highest degree of coincidence with the contact force simulation curve. Although the peak contact force calculated by piecewise nonlinear contact force lagged compared to simulation, but the peak contact force value, contact time and contact force-time curve are close to simulation. Therefore, the piecewise nonlinear contact force model can be used more precisely to calculate the contact force. The expression of piecewise nonlinear theoretical accelerated velocity based on the nonlinear contact force model is:

$$
\begin{aligned}
& a= \\
& \left\{\begin{array}{cl}
\frac{k_{1}}{m} y_{\Omega}(t)^{n} & \\
{\left[1+\frac{3\left(1-e_{h \Omega}\right) \dot{y}_{\Omega}(t)}{2 v_{0}}\right],} & y(t) \geq 0, \dot{y}(t)>0 \\
\frac{k_{2}}{m}\left(y_{\Omega}(t)-y_{\Omega}(0)\right)^{n} & \\
{\left[1+\frac{3\left(1-e_{h \Omega}\right) \dot{y}_{\Omega}(t)}{2 v_{0}}\right], c} & y(t) \geq y(0), \dot{y}(t)<0 \\
0, & \text { Others }
\end{array}\right.
\end{aligned}
$$

International Journal of Acoustics and Vibration, Vol. 25, No. 2, 2020 


\section{ANALYSIS OF CONTACT RESPONSE}

From the above research, contact response in the contact process can be predicted accurately by the piecewise nonlinear contact force model, the piecewise nonlinear accelerated velocity, the displacement model and velocity model obtained by the MSDOM and dimensionless parameter $\omega_{\Omega}$. Contact responses are different for conditions with different materials and contact parameters. In order to study the causes of the differences in contact response, we qualitative analyzed the impact of contact parameters and material properties on the contact response based on the optimal theoretical model under the working condition of elastic sphere which has similar properties to coal impacting the metal plate.

\subsection{Influence of Contact Parameters on Contact Response}

The varying of the contact parameters, such as contact damping and contact stiffness, will cause the change of the contact response. Fig. 12 shows the contact force, the displacement of the contact point, velocity and acceleration curves during the first collision process under different contact damping and different contact stiffness conditions.

With the increase of the contact stiffness $k_{1}$, the contact time and the maximum displacement at the collision contact point decrease, and the residual collision velocity, the maximum acceleration at the collision contact point increases. As a result, both the maximum contact force and the collision recovery coefficient increase. The above parameters all refer to the value, without considering the direction. As the increase of contact damping, the contact time is shortened, the maximum displacement of the contact point is gradually reduced, the residual collision velocity as well as the maximum acceleration of the collision contact point are both reduced, which result the maximum contact force and the collision recovery coefficient are diminished. The reason for the reduction of collision recovery coefficient with the increase of the damping ratio is that, because the initial velocity of the collision, the mass $m$, the radius $R_{1}$ and $k_{1}$ are invariant, so the $\omega_{\Omega}$ is invariant. Therefore, when the damping ratio $\xi$ increases, the damping $c$ increases, the damping force and energy dissipation of damping increase, then the system energy after the contact deformation process decreases, and the energy of the sphere in the rebound process decreases accordingly, which results in a reduction in the residual collision velocity. During the collision contact process, the contact deformation of the sphere decreases and the elastic recovery force increases with the contact stiffness $k_{1}$ increases, the wasted energy in the contact process decreases therewith it, then the sphere will bring more energy in the rebound process, as a result the residual collision velocity increases, so that the collision recovery coefficient increases.

\subsection{Influence of Material Properties on Contact Response}

The varying of the material properties can cause changes of the contact stiffness, which causes something different in contact response. The curves of the contact displacement, collision velocity, contact force, and impact acceleration are shown in Fig. 13 under conditions of unchanged damping $\mathrm{c}$ and changed density $\rho$, Poisson ratio $\nu_{1}$ and elastic modulus $E_{1}$ of the elastic sphere which had similar properties with coal. In this section, it also only researches the first collision contact.
With the increase of the density $\rho$, the contact time, the maximum contact force, the maximum contact displacement, the residual collision velocity and the collision recovery coefficient are all increase, the maximum acceleration decreases. The reason is that, the radius of sphere $R$ and other material properties are constant, the mass of the sphere $\mathrm{m}$ and the input initial energy (The contact energy of the sphere) of the system increase with the increase of the density $\rho$, the contact stiffness $k_{1}$ is constant, the energy absorbed by the elastic sphere in the end of the sphere compressed stage increases, so the maximum contact displacement (i.e. the maximum contact deformation of the sphere) increase. According to the piecewise nonlinear contact force model Eq. (26), the contact force increases, too.

With the increase of the Elastic Modulus $E_{1}$ and the Poisson's ratio $\nu_{1}$, the contact time and the maximum contact displacement both decrease, the maximum contact force, the residual collision velocity, the maximum acceleration and the collision recovery coefficient all increase. The reason is that, the contact stiffness $k_{1}$ increase with the increase of $E_{1}$ and $\nu_{1}$.

The curves of contact displacement, collision velocity, contact force and collision acceleration with the change of the Elastic Modulus $E_{2}$ and the Poisson's ratio $\nu_{2}$ are shown in Fig. 15. According to the Fig. 15, with the increase of the Elastic Modulus $E_{2}$, the contact time and the maximum contact displacement decrease, the maximum contact force, the residual collision velocity, the maximum acceleration and the collision recovery coefficient all increase. The reason is that the contact stiffness $k_{1}$ increase with the increase of $E_{2}$. As the Poisson's ratio $\nu_{2}$ changes, the first contact response of collision has little change, the reason is that: according to the expression of contact stiffness $k_{1}=\frac{4}{3}\left(\frac{1-\nu_{1}^{2}}{E_{1}}+\frac{1-\nu_{2}^{2}}{E_{2}}\right)^{-1} R_{1}{ }^{\frac{1}{2}}$ thanking to $E_{2}>>E_{1}>>\nu\left(\nu_{1}, \nu_{2}\right)$, compared with $\frac{1-\nu_{1}^{2}}{E_{1}}$, $\frac{1-\nu_{2}^{2}}{E_{2}}$ tends to 0 . Therefore, the influence of Poisson's ratio $\nu_{2}$ on contact stiffness is little, and there is almost no effect on collision contact process.

\section{CONCLUSIONS}

In this paper, by establishing the MSDOM, defining the dimensionless parameter $\omega_{\Omega}$, and combining with the finite element simulation of the elastic sphere impacting the rigid plate, the contact theory and contact response are analyzed. The following conclusions can be obtained that:

(1) The contact deformation (displacement of the centre of sphere), velocity, piecewise nonlinear contact force and acceleration can be used to calculate the contact response accurately based on the MSDOM and the dimensionless parameters $\omega_{\Omega}$.

(2) The peak contact force calculated by piecewise nonlinear contact force is lagged behind compared to simulation, but the peak contact force value, contact time and contact force-time curve are close to simulation.

(3) With the increase of contact stiffness, the maximum contact displacement decreases, the maximum contact force, the remaining impact velocity, the maximum acceleration increase and the coefficient of restitution increase. With the increase of contact damping, the maximum contact displacement gradually decreases. The maximum contact force, the remaining impact velocity, the maximum acceleration, and the coefficient of restitution decrease as well.

(4) As the density $\rho$ increases, the maximum acceleration 

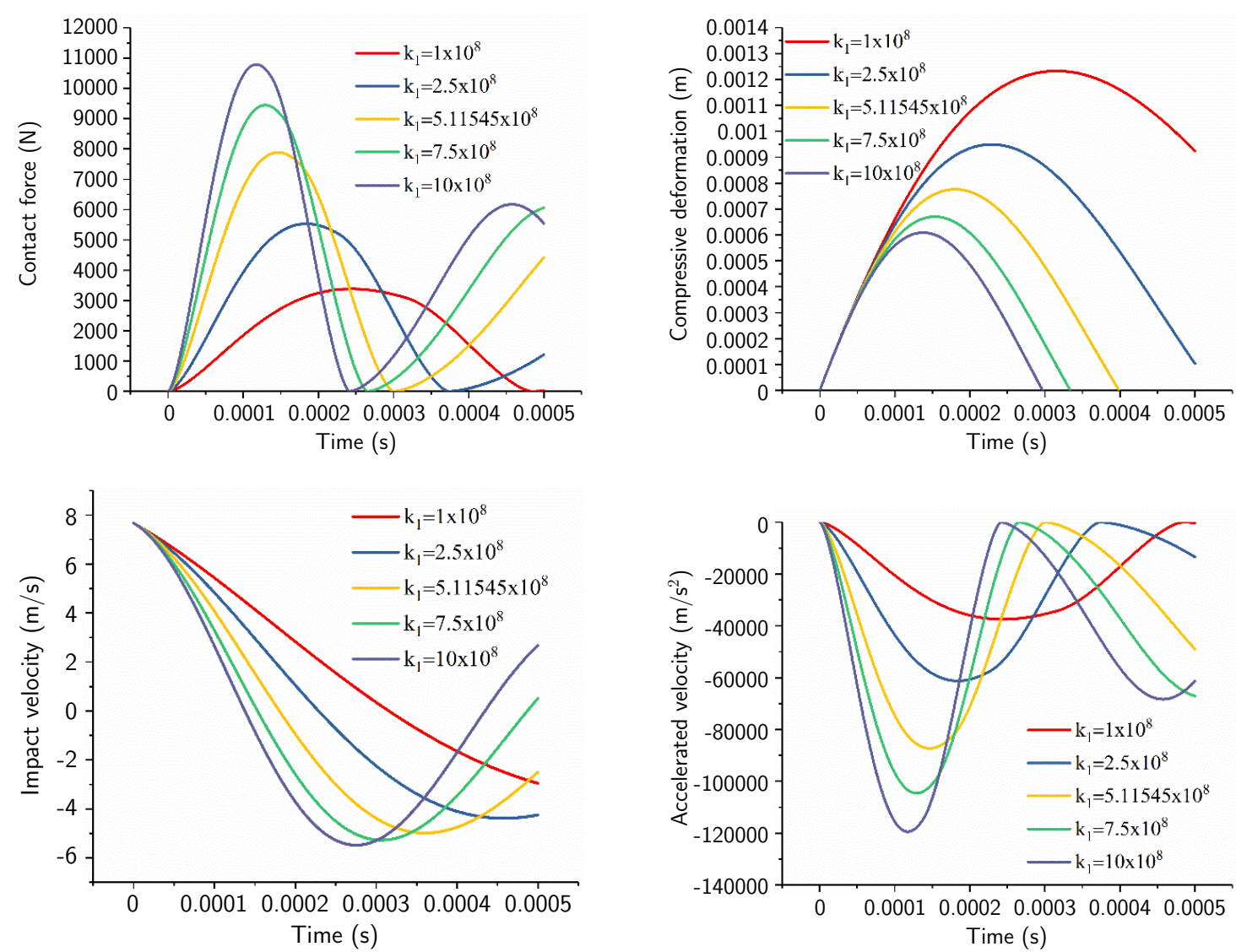

(1) Influence of contact stiffness on contact response
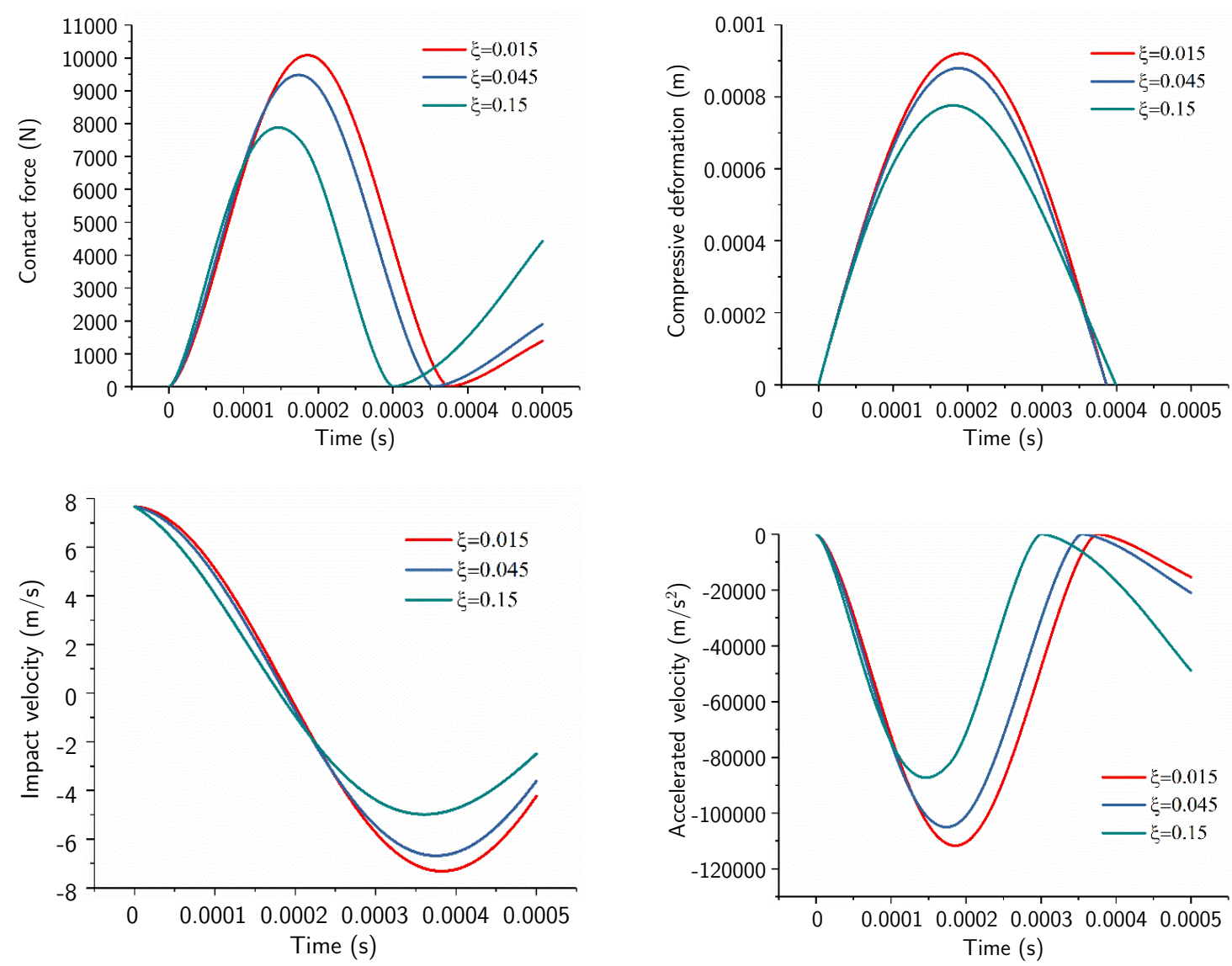

(2) Influence of contact damping ratio on contact response

Figure 12. Influence of contact parameters on contact response. 

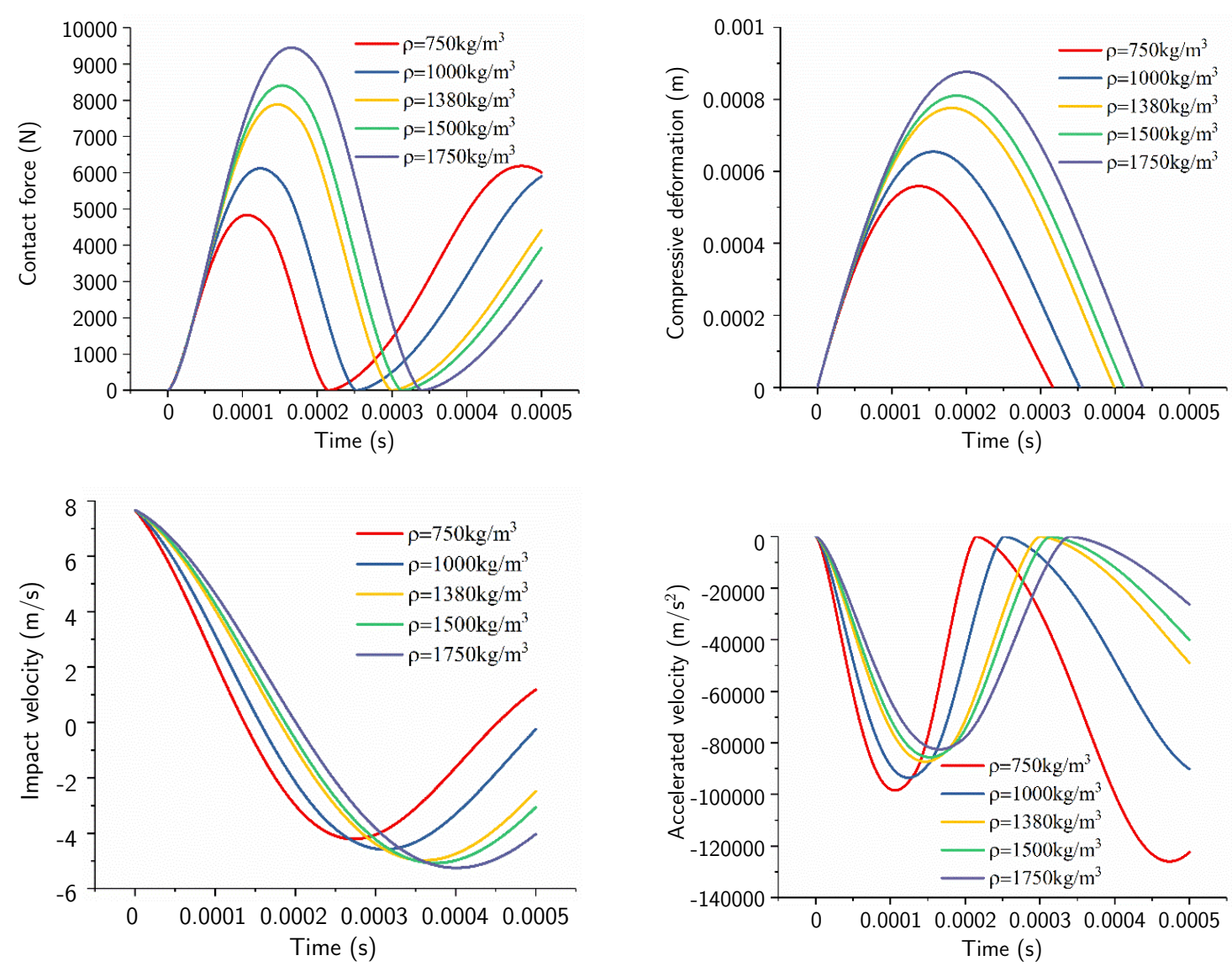

(1) Influence of sphere density $\rho$ on contact response
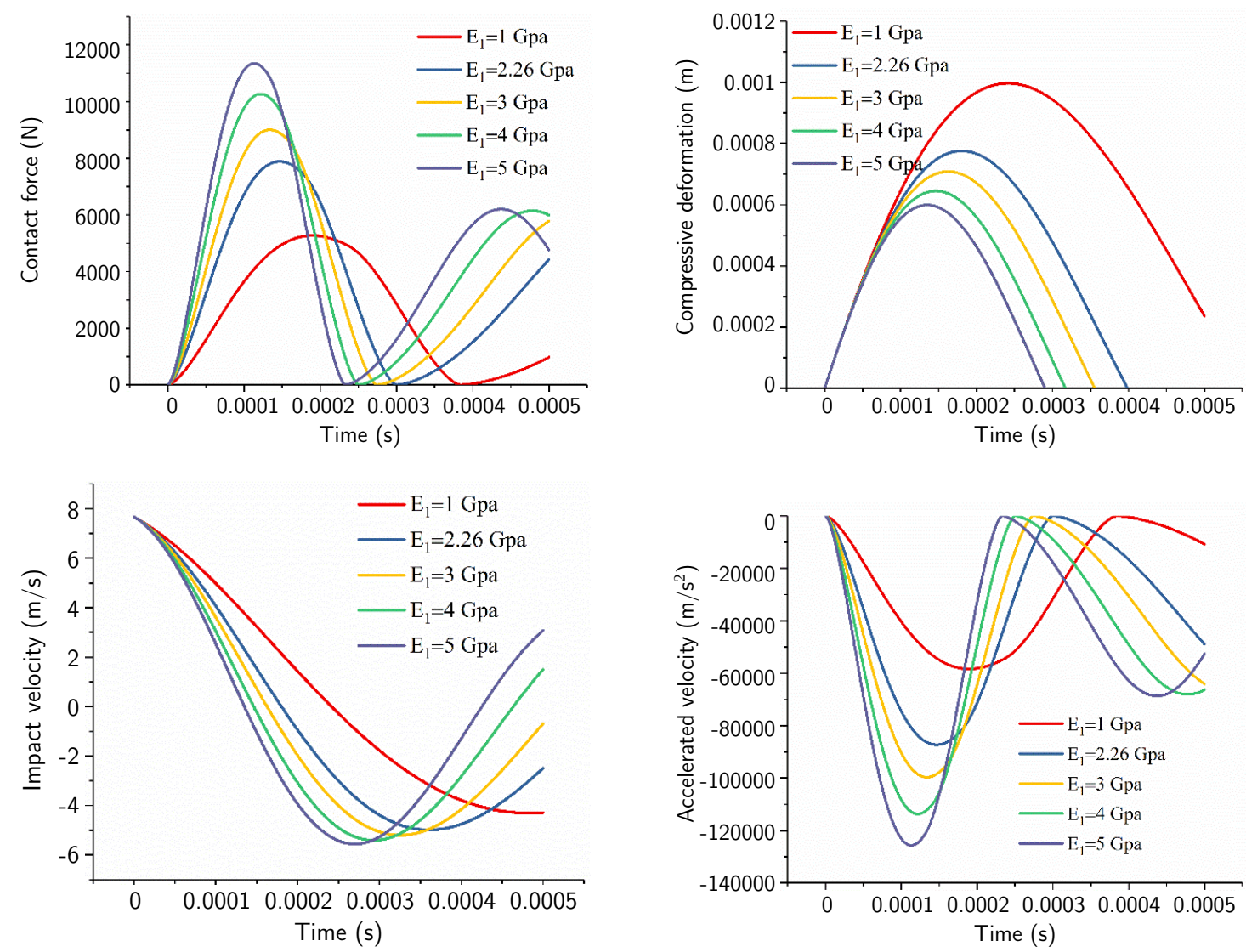

(2) Influence of sphere density $E_{1}$ on contact response

Figure 13. Influence of sphere's contact parameters on contact response. Continued on the next page. 


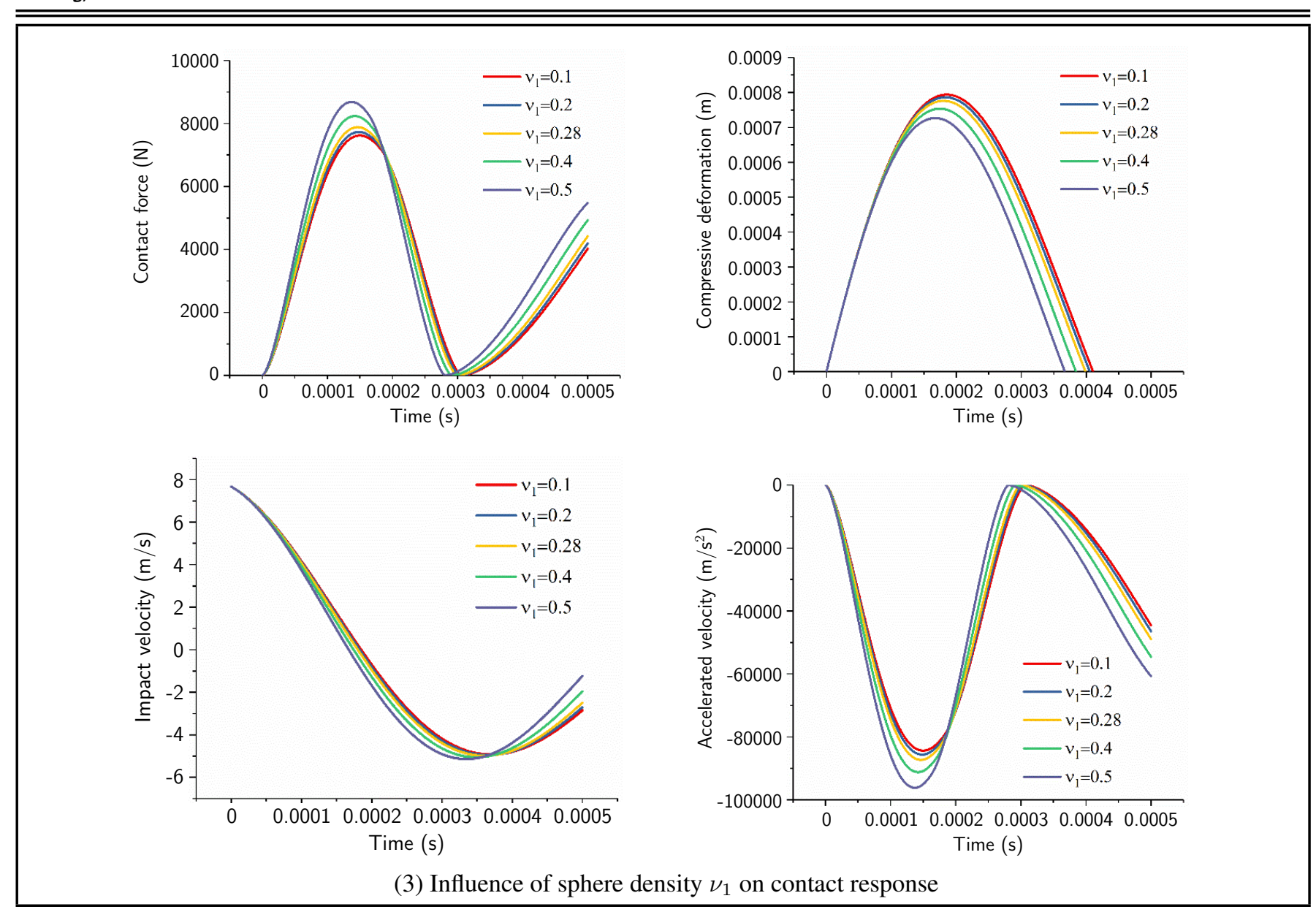

Figure 14. Influence of sphere's contact parameters on contact response. Continued from the previous page.

decreases, the maximum contact force, the maximum contact displacement, the remaining impact velocity and the coefficient of restitution increase. As the elastic modulus $E_{1}$ and $E_{2}$ and Poisson's ratio $\nu_{1}$ increase, the maximum contact displacement and the contact time decrease. Additionally, the maximum contact force, the remaining impact velocity, the maximum acceleration and the coefficient of restitution increase as well. Poisson's ratio $\nu_{2}$ has little effect on the first impact contact response.

This paper will provide a theoretical basis for impact contact study of elastic sphere impacting the rigid plate, and provide reference to contact study based on MSDOM and dimensionless parameters $\omega_{\Omega}$.

\section{ACKNOWLEDGEMENT}

This work supported by National Natural Science Fund of China (Grant No. 51674155), Innovative Team Development Project of Ministry of Education (Grant No. IRT_16R45), Special funds for Climbing Project of Taishan Scholars, Natural Science Foundation of Shandong Province (Grant No. ZR2016EEM09), and China Postdoctoral Science Foundation (Grant No.2016M602163).

\section{CONFLICTS OF INTEREST}

The authors declare that they have no conflict of interest concerning the publication of this manuscript.

\section{STATEMENT}

This manuscript has not been published, simultaneously submitted or already accepted for publication elsewhere. All authors have read and approved the manuscript. There is no conflict of interest related to individual authors' commitments and any project support. All acknowledged persons have read and given permission to be named. Yang Yang et al. have nothing to disclose.

\section{AUTHOR CONTRIBUTIONS}

Yang Yang and Qingliang Zeng established the new theory; Yang Yang and Lirong Wan performed the simulation, analysing the data; Yang Yang wrote the paper; Liang Wang and Guangjun Yin made advice for the article structure.

\section{REFERENCES}

1 Pham, T.M., Hao, Y.F. and Hao, H. Sensitivity of impact behaviour of RC beams to contact stiffness, International Journal of Impact Engineering, 112, 155-164, (2018). https://dx.doi.org/10.1016/j.ijimpeng.2017.09.015

2 Kharaz, A.H., Gorham, D.A. and Salman, A.D. An experimental study of the elastic rebound of spheres, Powder Technology, 120, 281-291, (2001). https://dx.doi.org/10.1016/S0032-5910(01)00283-2

3 Flores, P., Amabrósio, J. and Claro, J.P. Dynamic Analysis for Planar Multibody Mechanical Systems with Lubricated 

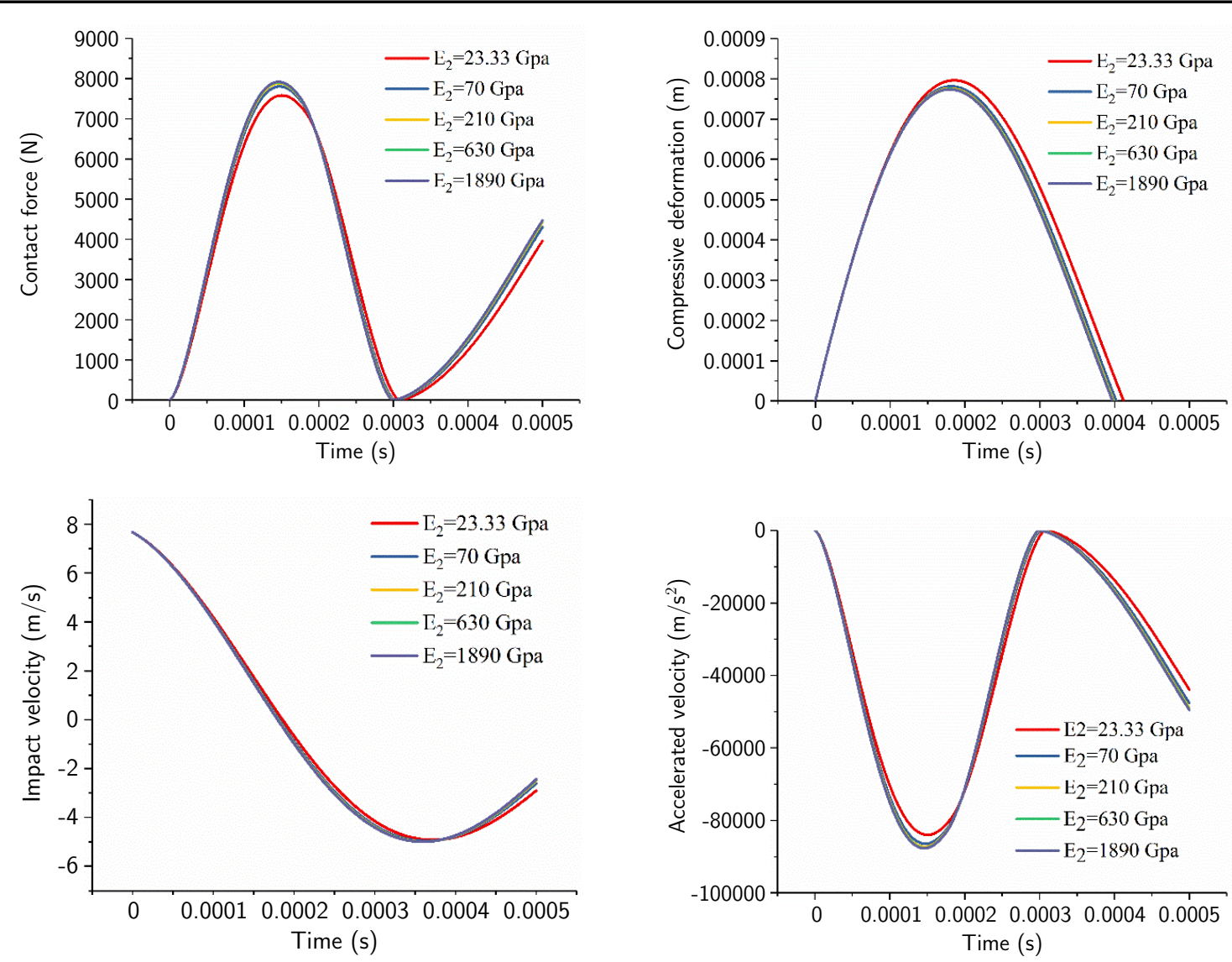

(1) Influence of sphere density $E_{2}$ on contact response
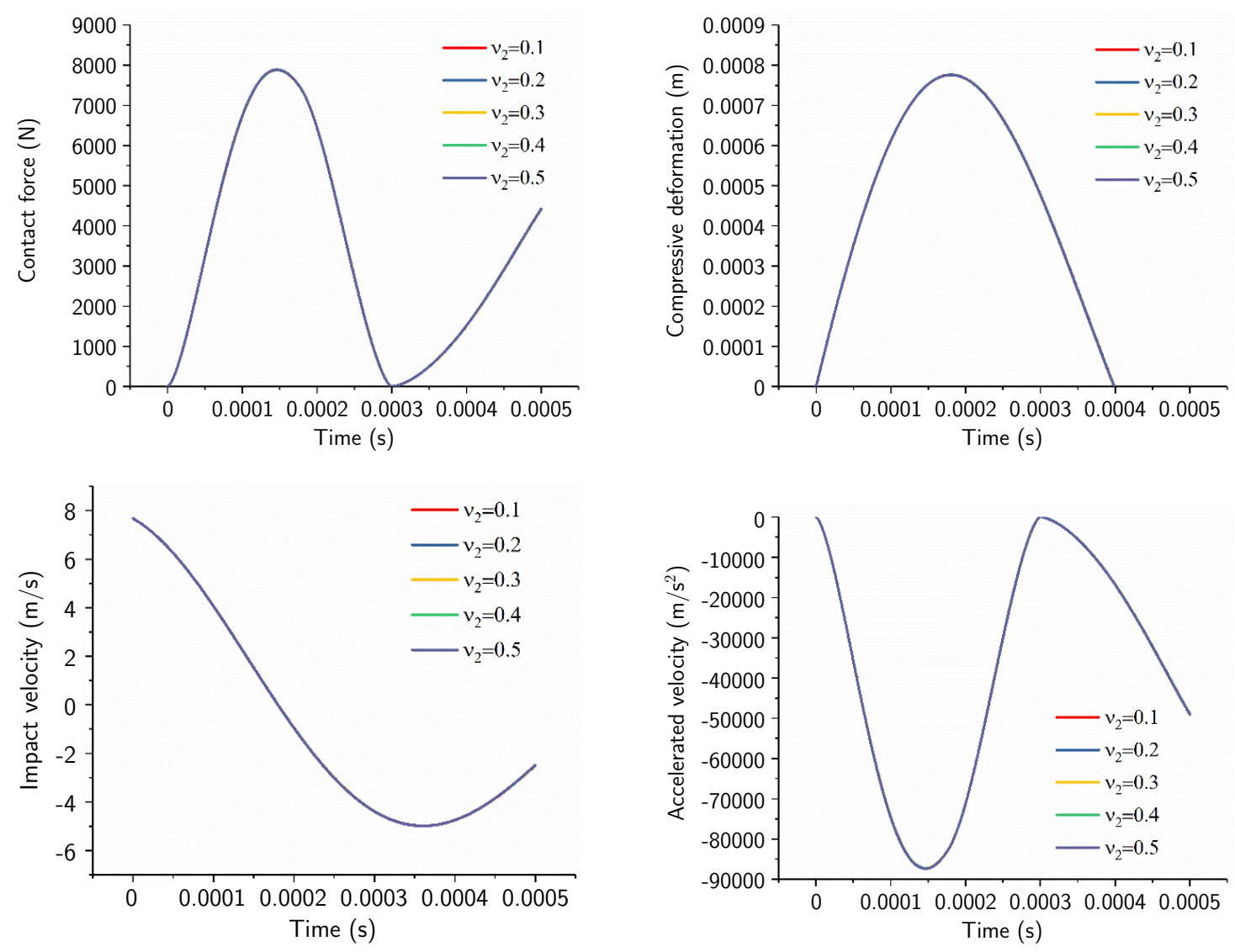

(2) Influence of sphere density $\nu_{2}$ on contact response

Figure 15. Influence of rigid plate's contact parameters on contact response. 
Joints, Multibody System Dynamics, 12, 47-74, (2004). https://dx.doi.org/10.1023/B:MUBO.0000042901.74498.3a

${ }^{4}$ Stronge, W.J. and Ashcroft, A.D.C. Oblique impact of inflated balls at large deflections, International Journal of Impact Engineering, 34, 1003-1019, (2007). https://dx.doi.org/10.1016/j.ijimpeng.2006.04.006

5 Du, Y.C. and Wang, S.L. Energy Dissipation in Normal Elastoplastic Impact Between Two Spheres. Journal of Applied Mechanics, 76 (6), 1089-1094, (2009). https://dx.doi.org/10.1115/1.3130801

${ }^{6}$ Goltsberg, R. and Etsion, I. Contact area and maximum equivalent stress in elastic spherical contact with thin hard coating, Tribology International, 93, 289-296, (2016). https://dx.doi.org/10.1016/j.triboint.2015.09.034

7 Braccesi, C. and Landi, L. A general elastic-plastic approach to impact analysis for stress state limit evaluation in ball screw bearings return system, International Journal of Impact Engineering, 34, 1272-1285, (2007). https://dx.doi.org/10.1016/j.ijimpeng.2006.06.005

${ }^{8}$ Brizmer, V., Kligerman, Y. and Etsion, I. The effect of contact conditions and material properties on the elasticity terminus of a spherical contact, International Journal of Solids and Structures, 43, 5736-5749, (2006). https://dx.doi.org/10.1016/j.ijsolstr.2005.07.034

9 Thornton, C., Ning, Z.M., Wu C.Y., Nasrullah, M. and Li, L.Y. Contact Mechanics and Coefficients of Restitution, Granular Gases, 564, 184-194, (2001). https://dx.doi.org/10.1007/3-540-44506-4_9

10 Thornton, C. Coefficient of Restitution for Collinear Collisions of Elastic-Perfectly Plastic Spheres, Journal of Applied Mechanics, 64 (2), 383-386, (1997). https://dx.doi.org/10.1115/1.2787319

${ }^{11}$ Hunt, K. and Crossley, E. Coefficient of restitution interpreted as damping in vibroimpact, Journal of Applied Mecha-nics, 42 (2), 440-445, (1975). https://dx.doi.org/10.1115/1.3423596

12 Lankarani, H.M. and Nikravesh, P. E. A contact force model with hysteresis dam-ping for impact analysis of multibody systems, Journal of Mechanical Design, 112 (3): 369-376, (1990). https://dx.doi.org/10.1115/1.2912617

${ }^{13}$ Lankarani, H.M. and Nikravesh, P. E. Continuous contact force models for impact analysis in multibody systems, Nonlinear Dynamics, 5 (2): 193-207, (1994). https://dx.doi.org/10.1007/BF00045676

${ }^{14}$ Flores, P., Machado, M., Silva M.T. and Martins, J. On the continuous contact force models for soft materials in multi-body dynamics, Multibody System Dynamics, 25, 357-375, (2011). https://dx.doi.org/10.1007/s11044-0109237-4

15 Bischoff, P. H., Perry, S. H. And Eibl, J. Contact Force Calculations With A Simple Spring-Mass Model For Hard Impact: A Case Study Using Polystyrene Aggregate Concrete. Journal of Impact Engineering, 9 (3), 317-325, (1990). https://dx.doi.org/10.1016/0734-743X(90)90005$\mathrm{G}$
${ }^{16}$ Qin, Z. Y. And Lu, Q. S. Analysis Of Impact Process Model Based On Restitution Coefficien, Journal Of Dynamics and Control, 14 (4), 294-298, (2006).

17 Mishra, B.K. A review of computer simulation of tumbling mills by the discrete element method:Part I-contact mechanics, Int. J. Miner. Process, 71, 73-93, (2003). https://dx.doi.org/10.1016/S0301-7516(03)00032-2

18 Xiao, H. F., Brennan, M. J. and Shao, Y. M. On the undamped free vibration of a mass interacting with a Hertzian contact stiffness. Mechanics Research Commu-nications 38, 560-564, (2011). https://dx.doi.org/10.1016/j.mechrescom.2011.07.012

19 Johnson, K. L. Contact mechanics [M], Cambridge University Press, (1985).

${ }^{20}$ LIU, Y., DU, C.L., FU, L. and GAO, K. D. Impact crushing velocity of lump coal, Journal of Vibration and Shock, 30 (3), 18-21, (2011). https://dx.doi.org/10.13465/j.cnki.jvs.2011.03.018

${ }^{21}$ Qin, Z.Y., Zhao, Y.J. and Hou S.J. Nonlinear Force Model During Mate-rial's Impact Crushing, Journal of Vibration and Shock, 25(2), 35-37+182, (2006). https://dx.doi.org/10.13465/j.cnki.jvs.2006.02.009

\section{APPENDIX A}

$$
\begin{gathered}
A_{1 \Omega}=\frac{g}{\omega_{\Omega}^{2}} ; \\
A_{2 \Omega}=\frac{\omega_{d \Omega} v_{0}-g \xi \sqrt{1-\xi^{2}}}{\omega_{d \Omega}^{2}} ; \\
\xi=\frac{c}{2 m \omega_{\Omega}}<1 ; \\
\omega_{d \Omega}=\sqrt{1-\xi^{2}} \omega_{\Omega} ; \\
B_{1 \Omega}=\sqrt{\left(g-2 \xi v_{0} \omega_{\Omega}\right)^{2}+\frac{\left(2 \xi^{2} v_{0} \omega_{\Omega}-g \xi-v_{0} \omega_{\Omega}\right)^{2}}{\left(1-\xi^{2}\right)}} ; \\
B_{2 \Omega}=\frac{\left(g-2 \xi v_{0} \omega_{\Omega}\right) \sqrt{1-\xi^{2}}}{2 \xi^{2} v_{0} \omega_{\Omega}-g \xi-v_{0} \omega_{\Omega}} ; \\
C_{1 \Omega}=\frac{1}{\omega_{\Omega}} \sqrt{\frac{g^{2}-2 g \xi v_{0} \omega_{\Omega}+v_{0}^{2} \omega_{\Omega}^{2}}{1-\xi^{2}}} ; \\
C_{2 \Omega}=\frac{g-\xi v_{0} \omega_{\Omega}}{v_{0} \omega_{\Omega} \sqrt{1-\xi^{2}}} ; \\
-\xi v_{0}^{2} \omega_{\Omega}^{2}-g^{2} \xi+2 g \xi^{2} v_{0} \omega_{\Omega} \\
D_{1 \Omega}=\frac{1}{\sqrt{1-\xi^{2}}\left(-v_{0}^{2} \omega_{\Omega}^{2}+2 g \xi v_{0} \omega_{\Omega}-g^{2}\right)} \\
\left.t_{c \Omega}=\frac{\pi}{\omega_{d \Omega}}+\arctan C_{2 \Omega}\right) .
\end{gathered}
$$

